\title{
Using Informal Conversation in the Clinical Setting as a Measure of Communication Potential for Adults with Aphasia
}

\author{
Kim C. McCullough \\ Department of Speech Language Pathology, University of Central Arkansas, Conway, 72035, United States of America
}

\begin{abstract}
The purpose of this study was to investigate the clinical usefulness of informal conversation as a tool for determining ability to communicate potential regardless of modality (verbal or nonverbal). Four individuals with aphasia (two non-fluent and two fluent) and four non-impaired individuals participated in this study. Selected segments of conversational discourse were analyzed for communication act usage during a 20-30 minute dyadic interaction with the investigator. Results revealed no significant differences between the total number of communication acts used by the participants. However, the participants with aphasia used a higher number of nonverbal and a combination of both verbal and nonverbal acts when compared to the non-impaired participants. Implications for clinical application are discussed .
\end{abstract}

Keywords Aphasia, Communication, Conversation, Verbal, Non-Verbal

\section{Introduction}

Given that communication is required for most daily activities, the impact of aphasia on functions of everyday life can be significant. Concepts of aphasia have been influenced by the World Health Organization's International Classification of Functioning, Disability, and Health (ICF). The ICF model provides clinicians with a framework for conceptualizing the development of treatment goals, not only for impairment and activity limitations but also for participation in life situations and social roles. The American Speech-Language-Hearing Association (ASHA) has recommended the use of the ICF model as outlined in the Scope of Practice in Speech-Language Pathology (ASHA, 2001) and Preferred Practice Patterns for the Profession of Speech-Language Pathology (ASHA, 2004). ASHA encourages clinicians to address impairments, activity limitations, and participation restrictions in their assessment and treatment of communication disorders. Additionally, ASHA recommends that clinicians recognize the environmental and personal factors that may have an influence on communication.

The ICF defines communication for societal participation as "holding a conversation: starting and sustaining an interchange of thoughts and ideas, carried out by means of spoken, written, sign or other forms of language, with one or

* Corresponding author:

kmccullo@uca.edu (Kim C. McCullough)

Published online at http://journal.sapub.org/health

Copyright (C) 2011 Scientific \& Academic Publishing. All Rights Reserved more people one knows or who are strangers in formal or casual settings" (WHO, 2001, p. 135). The strategic use of informal conversation is a readily available tool that clinicians can use as a way to develop treatment goals that will facilitate a person's potential for societal participation.

The present investigation used the concept of "communication acts" as a means for estimating communication potential. This investigation was designed to explore how individuals with aphasia circumvent their verbal deficits by using nonverbal strategies when expressing a communicative intent.

The results of previous investigations relating to usage of communication acts during conversation and aphasia suggest that communication acts may not necessarily be resistant to the breakdown that is seen in other aspects of the language of individuals with aphasia, as indicated by Holland (1982). Instead, it appears that some individuals with aphasia may exhibit a normal range of communication acts in comparison to non-impaired participants (Glosser et. al, 1988; Guilford \& O'Connor, 1982; Holland, 1982; Prinz, 1980), whereas others may display a restricted range of communication acts (Gurland, Chwat, \& Wollner, 1982; Prutting \& Kirchner, 1987; Wilcox \& Davis, 1977). One limitation of the above studies is that they fail to consistently report the extent to which individuals rely on nonverbal variables to communicate their message. Because nonverbal features (Damico et. al, 2008) are an inherent component of communicative interactions, inclusion of this data would provide a more accurate characterization of the ability of individuals with aphasia to effectively communicate intent. 
The purpose of this study was to compare the use of communication acts by individuals with aphasia and non-impaired individuals. An attempt was made to answer the following questions: 1) Is there an apparent difference in the number of communication acts used by individuals with aphasia and non-impaired individuals during informal conversation?; 2) Is there an apparent difference in the number of verbal and nonverbal communication acts used by individuals with aphasia and non-impaired individuals during informal conversation?; and 3) Is there an apparent difference in the distribution of usage across different types of communication acts that are expressed by individuals with aphasia and non-impaired individuals during informal conversation?

\section{Method}

\subsection{Participants}

Four individuals with aphasia (two fluent and two non-fluent) and four non-impaired controls participated in this study. All participants were recruited through local nursing homes.

The participants with aphasia were all pre-morbidly right-handed, native English speakers with a mean age of 71.25 years and a mean education level of 11.25 years. Participants were at least two months post-onset to insure medical stability. According to each participant's history, there was no evidence of neurologic disorder; psychiatric disorder; or speech, language, and hearing problems previous to the stroke.

The non-impaired participants were all pre-morbidly right-handed, native English speakers with a mean age of 72.5 years and a mean education level of 10.75 years. According to each participant's history, there was no evidence of neurologic disorder; psychiatric disorder; or speech, language, and hearing problems. The non-impaired participants were matched in age, gender, and education with the participants with aphasia. Two females and two males were recruited for each participant group. Table 1 summarizes information obtained for each participant.

Table 1. Descriptive Information of Participants

\begin{tabular}{ccccc}
\hline Group & $\begin{array}{c}\text { Age } \\
\text { (Years) }\end{array}$ & $\begin{array}{c}\text { Education } \\
\text { (Years) }\end{array}$ & $\begin{array}{c}\text { Time Post } \\
\text { CVA } \\
\text { (mos.) }\end{array}$ & Gender \\
\hline $\begin{array}{c}\text { P\#1 } \\
\text { Non-fluent }\end{array}$ & 74 & 14 & 27 & $\mathrm{M}$ \\
P\#2 Fluent & 73 & 13 & 38 & $\mathrm{~F}$ \\
P\#3 & 66 & 12 & 4 & $\mathrm{~F}$ \\
Non-Fluent & $\underline{74}$ & $\underline{4}$ & $\underline{13}$ & $\mathrm{M}$ \\
P\#4 Fluent & 71.75 & 10.75 & 20.50 & \\
$\underline{\mathrm{M}}$ & 76 & 14 & & $\mathrm{M}$ \\
Control 1 & 74 & 11 & & $\mathrm{~F}$ \\
Control 2 & 66 & 12 & & $\mathrm{~F}$ \\
Control 3 & $\underline{74}$ & $\underline{8}$ & & $\mathrm{M}$ \\
Control 4 & 72.50 & 11.25 & & \\
M & & & &
\end{tabular}

\subsection{Preliminary Testing}

The Western Aphasia Battery (WAB) (Kertesz, 1982) was administered to all participants. As expected, the non-impaired participants presented with language skills that were judged to be within normal limits. Individual scores from the WAB are presented in Table 2. All participants participated in a hearing screening and hearing was judged to be adequate for conversation.

\subsection{Experimental Task}

The primary objective of the experimental task was to elicit a spontaneous communication sample that was representative of each participant's ability to communicate regardless of modality. Therefore, it was necessary to embed this portion in such a way that the participants were not self-conscious or poised for the communication sample. Previous literature suggests that this is possible by first administering formal assessments and then suggesting that a "break" from testing be taken (Ripich, Vertes, Whitehouse, Fulton, \& Ekelman, 1991).

Each experimental session lasted approximately two hours and took place in a clinic treatment room with the participant and investigator seated across the table from one another. After the administration of the WAB and the hearing screening, each participant was told that it was time for a coffee break from testing. Coffee and a light snack were then offered to the participant. At this point, the investigator engaged the participant in a 20-30 minute informal conversation. Work history and illness were targeted for topics of conversation because they have been found to yield the most complex language (Glosser et al., 1988). Alternative conversational topics included information relating to recent activities and interests, recent life changes, education and family. The investigator attempted to keep the topics and the form of the conversations similar for all participants. However, the content of the conversations depended largely on the participant. All interactions were videotape recorded. The participant's communication acts within fifty investigator communication acts after an initial two minute period of talking were used for analysis. The mode of communication was also analyzed. This included verbal, nonverbal, and combinations of verbal and nonverbal modes.

\subsection{Data Analysis}

The choice for system of analysis was determined based on the communication categories used in previous research with adults (Doyle, Thompson, Oleyar, \& Wambaugh, 1994; Gurland et al., 1982; Ripich et al., 1991; Wambaugh et al., 1991; Wilcox \& Davis, 1977). Dore's (1978) Conversational Act Categories was chosen for the system of analysis because it included many of the same categories used in the above mentioned adult studies. The following communicative act categories were analyzed during the conversation (Dore, 1978): requests, responses to requests, descriptions, statements, acknowledgments, organizational devices, performatives, and miscellaneous. 
Table 2. Individual Participant Scores on Western Aphasia Battery

\begin{tabular}{cccccc}
\hline Participant & $\begin{array}{c}\text { Spontaneous } \\
\text { Speech }\end{array}$ & Comprehension & Repetition & Naming & $\begin{array}{c}\text { Diagnostic } \\
\text { Category }\end{array}$ \\
\hline P\#1 Non-fluent & 4 & 2.85 & 4.2 & 1.4 & Broca \\
P\#2 Fluent & 14 & 5.7 & 1.1 & 2.9 & Wernicke \\
P\#3 Non-fluent & 10 & 4.7 & 1.8 & 3.1 & Broca \\
P\#4 Fluent & 6 & 1.4 & 0.0 & 0.0 & Wernicke \\
& & & & 9.5 & Non-impaired \\
Control 1 & 20 & 10 & 10 & 9.3 & Non-impaired \\
Control 2 & 20 & 10 & 10 & 9.1 & Non-impaired \\
Control 3 & 20 & 10 & 10 & 8.5 & Non-impaired \\
Control 4 & 18 & 9.9 & 10 & & \\
\hline
\end{tabular}

Note. Maximum score for Spontaneous Speech $=20$, Comprehension $=10$, Repetition $=10$, Naming $=10$.

Table 3. Examples of Nonverbal Behaviors Considered as Expressions of Communicative Acts

\begin{tabular}{cc}
\hline Behaviors & Explanation \\
\hline Intensity, Rate, Pitch & Vocal effects which accompany the linguistic message \\
Facial Expression & Eye contact, lip movement \\
Gesture & Manual movements or silent acting where speech is replaced, for example, by raising an imaginary cup \\
Body Posture & Manner in which subject sits \\
Head Movement & Side-to-side and back-to-front movements \\
\hline
\end{tabular}

Note. Adapted from "Non-verbal Communication of Aphasic Patients," by M. Behrmann and C. Penn, 1984, British Journal of Communication, 19, p. 158.

The principal investigator developed written transcripts, using English orthography, from the videotapes to capture all verbal and nonverbal expressions of the communication acts.

The principal investigator initially classified each expression as verbal, nonverbal or a combination of both verbal and nonverbal. See Table 3 for a description of nonverbal behaviors that were considered to be communicative. The primary investigator then coded each communicative expression in terms of its communication act using Dore's (1978) categories. The investigator's communication acts were coded to provide information regarding the context of the communication acts and the intent expressed by the participants.

\subsection{Reliability}

For the purposes of intra-judge reliability of transcription, the primary investigator selected at random one language sample from each group of participants. The videos were viewed and discrepancies in transcription from the original transcripts were noted. The percent agreement for intra-reliability of the two randomly selected transcripts was $98 \%$.

For the purposes of inter-judge reliability of transcription, two language samples were selected at random from each group. A trained reviewer viewed the videotapes of the four samples and generated an independent transcription for each sample. The reviewer was then given the original transcriptions generated by the principal investigator and instructed to indicate any discrepancies between the transcriptions. The percent agreement for the four randomly selected transcripts was $89 \%$. Points of disagreement were then identified and discussed, and agreement was reached on all points.

For the purposes of intra-judge reliability of coding of communication acts, the primary investigator re-coded ten percent of a randomly selected portion of the communication acts for each of the eight participants. The percent agreement for this procedure was $97 \%$.

For the purposes of inter-judge reliability of coding, twenty-five percent of the communication acts from each of the eight original transcripts were randomly selected for re-coding by the trained reviewer. Point-to-point agreement was $98 \%$. All points of disagreements were identified, the transcripts and videotapes were again viewed by the primary investigator and trained reviewer and, after discussion, agreement was reached on all points.

\subsection{Results}

Because of the small sample size parametric statistical analyses were not performed; however, individual and group means were analyzed to determine trends in communication act usage. As stated previously, the number of communication acts for each participant was tabulated. The mode of each communication act was classified as verbal, nonverbal or a combination of both and each communication act was coded using Dore's (1978) categories.

Table 4. Summary of Total Number of Communication Acts Used

\begin{tabular}{cc}
\hline Participant & Total Communication Act Usage \\
\hline P\#1 Non-fluent & 41 \\
P\#2 Fluent & 50 \\
P\#3 Non-Fluent & 32 \\
P\#4 Fluent & $\underline{42}$ \\
M (SD) & $41.25(7.37)$ \\
Control 1 & 50 \\
Control 2 & 45 \\
Control 3 & 41 \\
Control 4 & $\underline{57}$ \\
M (SD) & $48.25(6.90)$ \\
\end{tabular}


Table 5. Summary of Means and Standard Deviations for Mode of Communication

\begin{tabular}{cccc}
\hline Participants & Verbal \# & Nonverbal \# & Combination \# \\
\hline P\#1 Non-fluent & 6 & 14 & 21 \\
P\#2 Fluent & 34 & 0 & 16 \\
P\#3 Non-Fluent & 11 & 5 & 16 \\
P\#4 Fluent & $\underline{9}$ & $\underline{13}$ & $\underline{20}$ \\
$\underline{\mathrm{M}}$ & 15.00 & 8.00 & 18.25 \\
$\underline{\mathrm{SD}}$ & 12.83 & 6.68 & 6.68 \\
Control 1 & 43 & 0 & 7 \\
Control 2 & 27 & 0 & 18 \\
Control 3 & 36 & 2 & 6 \\
Control 4 & $\underline{50}$ & $\underline{1}$ & $\underline{6}$ \\
$\underline{\mathrm{M}}$ & 39.00 & 0.75 & 8.50 \\
$\underline{\mathrm{SD}}$ & 9.83 & 0.96 & 6.56 \\
\hline
\end{tabular}

Table 6. Summary of Percentage of Usage for Mode of Communication

\begin{tabular}{cccc}
\hline Participants & Verbal \% & Nonverbal \% & Combination \% \\
\hline P\#1 Non-fluent & 14.6 & 34.0 & 51.2 \\
P\#2 Fluent & 68.0 & 0.0 & 32.0 \\
P\#3 & 34.3 & 15.6 & 50.0 \\
Non-Fluent & & & \\
$\underline{\text { P\#4 Fluent }}$ & $\underline{21.4}$ & $\underline{30.9}$ & $\underline{47.6}$ \\
$\underline{\mathrm{M} \%}$ & 34.6 & 19.4 & 44.2 \\
Control 1 & 86.0 & 0.0 & 14.0 \\
Control 2 & 60.0 & 0.0 & 40.0 \\
Control 3 & 81.8 & 4.5 & 13.6 \\
Control 4 & $\underline{87.7}$ & $\underline{1.8}$ & $\underline{10.5}$ \\
M\% & 80.4 & 2.0 & 18.8 \\
\hline
\end{tabular}

Participants with aphasia produced a mean number of 41.25 communication acts and the non-impaired participants produced a mean number of 48.25 communication acts. A summary of the mean number of communication acts produced is presented in Table 4. Given these results there appears to be no apparent difference in the number of communication acts used by individuals with aphasia and non-impaired individuals during informal conversation.

The control participants had a mean of 3.25 for requests, 17.50 for responses, 14.75 for descriptions, 11.25 for statements, 3.0 for acknowledgments, .50 for organizational devices, .25 for performatives, and .50 for miscellaneous communication acts. Refer to Figure 5 for a visual display of these results.

Given these results there appears to be an apparent difference in the distribution of usage across the different types of communication acts expressed by the individuals with aphasia and the non-impaired individuals during informal conversation.

The investigator's communication acts were also coded to provide information regarding the context of the communication acts expressed by the participants. For the participants with aphasia, the investigator produced a mean percentage of $60.5 \%$ for requests, $3.5 \%$ for responses to requests, $8 \%$ for descriptions, $9 \%$ for statements, $18.5 \%$ for acknowledgments, and $0.5 \%$ for organizational devices.

For the control participants, the investigator produced a mean percentage of $38 \%$ for requests, $4 \%$ for responses to requests, $5.5 \%$ for descriptions, $13 \%$ for statements, and $38.5 \%$ for acknowledgments. See Table 8.

\subsection{Discussion}

Clinicians are challenged with the task of identifying preserved language areas and using these areas as starting points for initiating treatment. If specific functions are to be targeted for treatment, consideration should be given to how frequently these functions are normally used in conversation. It is for this reason that identification of patterns of performance among individuals with aphasia is important. The present study sought to determine the number, mode of expression, and variety of communication acts used by participants with aphasia when compared to non-impaired participants during informal conversation.

Visual inspection of the data revealed no apparent differences in the number of communication acts produced by the participants with aphasia when compared to the control participants. This finding lends support to research findings (Prutting \& Kirchner, 1987) that individuals with aphasia are sensitive to the social and interactive rules of conversation.

It was hypothesized that the participants with aphasia might use nonverbal strategies or a combination of verbal and nonverbal strategies to more effectively communicate a message. Results relating to mode of communication revealed an overall trend for the participants with aphasia to use more nonverbal strategies to communicate intent than the control participants. However, individual variability among the participants with aphasia was observed.

Table 7. Summary of Means and Standard Deviations for Communication Acts Usage by the Participants with Aphasia and Control Participants

\begin{tabular}{ccccccccc}
\hline Participants & $\mathrm{R}$ & $\mathrm{RR}$ & $\mathrm{D}$ & $\mathrm{S}$ & $\mathrm{A}$ & $\mathrm{OD}$ & $\mathrm{P}$ & $\mathrm{M}$ \\
\hline P\#1 Non-fluent & 0 & 35 & 0 & 1 & 5 & 0 & 0 & 0 \\
P\#2 Fluent & 2 & 20 & 22 & 4 & 1 & 0 & 0 & 1 \\
P\#3 Non-Fluent & 1 & 21 & 3 & 0 & 6 & 0 & 0 & 2 \\
P\#4 Fluent & $\underline{2}$ & $\underline{37}$ & $\underline{0}$ & $\underline{0}$ & $\underline{1}$ & $\underline{0}$ & $\underline{0}$ & $\underline{2}$ \\
$\underline{\mathrm{M}}$ & 1.25 & 28.50 & 6.25 & 1.25 & 3.25 & 0 & 0 & 1.25 \\
$\underline{\mathrm{SD}}$ & 0.96 & 9.0 & 10.59 & 1.89 & 2.63 & 0 & 0 & 0.96 \\
Control 1 & 6 & 17 & 16 & 7 & 2 & 1 & 0 & 1 \\
Control 2 & 2 & 27 & 0 & 13 & 2 & 0 & 0 & 0 \\
Control 3 & 1 & 14 & 28 & 18 & 2 & 1 & 0 & 1 \\
Control 4 & $\underline{4}$ & $\underline{12}$ & $\underline{15}$ & $\underline{7}$ & $\underline{6}$ & $\underline{0}$ & $\underline{1}$ & $\underline{0}$ \\
$\underline{\mathrm{M}}$ & 3.25 & 17.50 & 14.75 & 11.25 & 3.00 & 0.50 & 0.25 & 0.50 \\
$\underline{\text { SD }}$ & 2.22 & 6.66 & 11.47 & 5.32 & 2.00 & 0.58 & 0.50 & 0.58 \\
\hline
\end{tabular}

Note. $\mathrm{R}=$ Request, $\mathrm{RR}=$ Response to Request, $\mathrm{D}=$ Description, $\mathrm{S}-$ Statement, $\mathrm{A}=$ Acknowledgement, $\mathrm{O}=$ Organizational Device $\mathrm{P}=$ Performative, and $\mathrm{M}=$ Miscellaneous. 
Table 8. Summary of Percentage of Communication Acts Produced by the Investigator During Informal Conversation with the Participants with Aphasia and Control Participants

\begin{tabular}{|c|c|c|c|c|c|c|c|}
\hline Participants & $\mathbf{R}$ & $\mathbf{R R}$ & D & $\mathbf{S}$ & $\mathbf{A}$ & OD & $\mathbf{M}$ \\
\hline P\#1 Non-fluent & 72 & 0 & 2 & 8 & 18 & 0 & 0 \\
\hline P\#2 Fluent & 46 & 4 & 4 & 10 & 36 & 0 & 0 \\
\hline P\#3 Non-Fluent & 42 & 4 & 22 & 14 & 16 & 2 & 0 \\
\hline P\#4 Fluent & $\underline{81}$ & $\underline{6}$ & $\underline{4}$ & $\underline{4}$ & $\underline{4}$ & $\underline{0}$ & 0 \\
\hline$\underline{\mathrm{M}}$ & $60.5 \%$ & $3.5 \%$ & $8.0 \%$ & $9.0 \%$ & $18.5 \%$ & $0.0 \%$ & $0.0 \%$ \\
\hline Control 1 & 38 & 10 & 8 & 8 & 28 & 0 & 4 \\
\hline Control 2 & 32 & 2 & 4 & 4 & 54 & 0 & 0 \\
\hline Control 3 & 28 & 0 & 4 & 4 & 42 & 0 & 0 \\
\hline Control 4 & $\underline{54}$ & $\underline{4}$ & $\underline{6}$ & $\underline{6}$ & $\underline{30}$ & $\underline{0}$ & $\underline{0}$ \\
\hline$\underline{\mathrm{M}}$ & $38.0 \%$ & $4.0 \%$ & $13.0 \%$ & $13.0 \%$ & $38.5 \%$ & $0.0 \%$ & $1.0 \%$ \\
\hline
\end{tabular}

As reported in previous investigations (Guilford \& O'Connor, 1982), the degree and type of communicative deficit in the participants with aphasia appeared to have a direct influence on the type of communicative strategy utilized. For example, the participants with non-fluent aphasia tended to rely more on a combination of both verbal and nonverbal strategies to communicate intent. Both participants with non-fluent aphasia used the combination mode of expression for at least $50 \%$ of the communication acts they produced. These participants appeared to be more aware of their verbal impairments. The participants with non-fluent aphasia attempted to compensate for their deficits by using gestures and head nods to clarify and augment the verbal message put forth. Both the participants with non-fluent aphasia appeared to be more certain of the nonverbal information they were expressing and often revised the verbal information to match the nonverbal information provided.

In contrast to the participants with non-fluent aphasia, a significant amount of variability was observed between the two participants with fluent aphasia. The degree of communicative deficit between these two participants was quite different and appeared to have a direct influence on the type of communicative strategy that each utilized. Participant with aphasia \#4, who had a severe fluent aphasia, presented with speech that is best described as being fluent jargon, with little or no real words produced. Participant with aphasia \#2, who had a moderate fluent aphasia, presented with speech that did contain real words which enabled the investigator to interpret her verbal message more accurately than the more severely impaired fluent participant's. The severely impaired participant with fluent aphasia demonstrated a profile similar to the mode of communication of the participants with non-fluent aphasia. This participant used the nonverbal and combination mode of expression for $80 \%$ of the communication acts he expressed. The nonverbal strategies employed by the severely impaired participant with fluent aphasia were critical in his ability to express an intention. However, he was not able to revise his verbal expressions to match his nonverbal expressions. The moderately impaired participant with fluent aphasia employed more verbal strategies to communicate her intents. This participant used nonverbal behaviors only as a supplement to her verbal message. In this regard, her mode of communication act usage resembled that of the control participants.
In contrast, the function of the nonverbal communication acts used by the participants with aphasia differed from that of the control participants. The control participants used primarily verbal means to communicate an intention. Occasionally, a control participant would supplement the verbal message with a hand gesture, head nod, or change of into

nation. However, the nonverbal behaviors used by the control participants only complemented the verbal message produced and was not crucial in interpreting the intent.

The distribution of communication acts expressed by the participants with aphasia was limited in comparison to the control participants. This finding supports previous investigations (Gurland et al., 1982; Prutting \& Kirchner, 1987; Wilcox \& Davis, 1977) which report that individuals with aphasia exhibit a restricted variety of communication acts. It is important to emphasize that the results from the present study were obtained despite the fact that both the verbal and nonverbal modes were awarded communicative value. The participants with aphasia primarily produced responses to requests for information. For example, the investigator requested information from the participants with aphasia approximately $60 \%$ of the time whereas she only requested information from the control participants $38 \%$ of the time. The fluent participants differed greatly from each other in regards to the variety of usage of communication acts. The severely impaired participant with fluent aphasia, for the most part, only produced responses to requests for information from the investigator. In contrast, the moderately impaired participant with fluent aphasia was observed to use descriptions and statements more frequently.

Several possible variables may account for the limited usage observed. First, it appears that the degree and type of communicative deficit not only influences the mode of communication used but also the variety of communication acts produced by each participant. Several previous studies (Guilford \& O'Connor, 1982; Behrmann \& Penn, 1984) support the findings of the present study which indicate an influence of degree and type of deficit on the usage of communication acts.

For example, both the participants with non-fluent aphasia appeared to be aware of their verbal deficits. This awareness may in part be responsible for their limited amount of "talking". Informal observations of these participants with their caregivers revealed the same type of communication 
profile. These participants were not observed to initiate conversations or "talking", however, they did initiate communication when attempting to make a need known. For example, Participant \#1, an individual with non-fluent aphasia, was able to point to the door indicating to his wife that he wanted to go outside.

Another important variable to consider is the investigator's patterns of communication acts. It appeared that the investigator was retaining the clinician role of trained interviewer during the informal conversation. As stated previously, the investigator requested information from the participants with aphasia $60 \%$ of the time and only requested information from the control participants $38 \%$ of the time. Other researchers (Doyle et al., 1994; Wilcox \& Davis, 1977) have reported similar patterns of communication acts usage with clinicians. The investigator appeared to compensate for the communicative deficits of their participants with aphasia by requesting information and changing topics to extend the conversation. This structured and assistive mode of interaction adopted by the investigator during conversations with the participants with aphasia may have inhibited occurrence of, for example, requests or statements.

Furthermore, because the participants with aphasia had all received prior speech therapy services and were accustomed to having the clinicians structure the environment, it seemed likely that they either expected, or possibly allowed, the investigator to continue to structure the verbal environment. Additionally, the participants had completed an hour of testing with the investigator just before the informal conversation which may have also contributed to their limited usage of communication acts.

Visual inspection of the transcripts revealed that the non-impaired participants provided the investigator with a significantly larger amount of new information as compared to the participants with aphasia. Although the topics of conversation were relatively the same for the group of participants with aphasia and the control group, the control participants were able to answer requests for information with more detail and specific information. Although a mean length of utterance was not measured, visual inspection revealed the control participants' utterances to be much longer than those of the participants with aphasia. The control participants demonstrated further elaboration of responses and a greater proportion of total words per conversation when compared to the group of participants with aphasia as a whole. The non-impaired participants were able to develop and extend dialogue on specific topics as opposed to the shorter, less elaborated discussions that took place with the participants with aphasia, especially the participants with non-fluent aphasia. In the case of the participants with aphasia, their responses provided only the required information. For example, when asking if a participant with aphasia had children, the response was usually "yes". In contrast, the response to this question by the control participants was usually similar to "yes, I have three children. One lives in Florida and the other two live in Texas". The participants with aphasia were also observed to have more difficulty initiating and sustaining the conversation.

\section{Conclusions}

Results of this research were that individuals with aphasia produce the same overall number of communication acts, although the variety of communication acts was restricted when compared to non-impaired individuals. Furthermore, consideration of nonverbal behaviors used by individuals with aphasia during communication did not influence the findings as expected. It is of interest to note that the scores of the participants with aphasia on the WAB did not necessarily predict which participants would communicate most effectively during the informal conversation. Further research is needed to explore whether the standardized functional communication measures that are available might be a better predictor of the communication act usage of individuals with aphasia. It is anticipated that further investigation into the pragmatic abilities of individuals with aphasia will lead to information which may enhance the design of assessment and therapy that target full-life participation for this population.

Although the number of participants with aphasia included in this study was small, it appears that communication act usage may be related to severity of aphasic impairment and in particular, severity of verbal impairment. Further research is needed to explore performance profiles in both individuals with aphasia and non-impaired individuals.

Although specific information obtained from this study does not contribute directly to the development of appropriate treatment strategies for individuals with aphasia, these findings suggest that there may be distinctions among some individuals with aphasia which deserve attention, and which may ultimately enhance the design of clinical treatment for specific patients, given further exploration.

The idea of using a less impaired or relatively unaffected modality to strengthen an affected one is not a new one in the aphasia literature. It seems that the best way to implement this notion is to start providing opportunities in treatment sessions to produce a variety of communication acts. This does not mean to suggest that every treatment session should provide opportunities to communicate all possible intentions, for this is neither possible nor realistic. But rather, treatment should be structured so that the patient is allowed to intend more than a response to a clinician's request.

To understand how or whether clinical deficits affect communicative participation, analysis of larger segments of performance is necessary. These results suggest that the pragmatic aspects of language are closely linked to judgments of a perceived level of social competence. Our effectiveness as clinicians is judged, in part, by the impact our remediation efforts have on an individual's ability to function as a productive member of society (Kagan \& Simmons-Mackie, 2007). In cases where only limited advancement in the structural aspects of language can be pre- 
dicted, remediation of the pragmatic aspects of communication may contribute most to a level of social acceptability and participation.

\section{REFERENCES}

[1] American Speech-Language-Hearing Association (2001. Scope of practice in speech-language pathology. Available from www.asha.org/policy

[2] American Speech-Language-Hearing Association. (2004). Preferred practice patterns for the profession of speech-language pathology. Available from www.asha.org/policy

[3] World Health Organization. (2001). International Classification of functioning, disability and health. Geneva, Switzerland: Author

[4] Holland, A. L. (1982). Observing functional communication of aphasic adults. Journal of Speech and Hearing Disorders, $47,50-56$

[5] Glosser, C., Weiner, M., \& Kaplan, E. (1988). Variation in aphasic language behaviors. Journal of Speech and Hearing Disorders, 53, 115-124

[6] Guilford, A. M., \& O'Connor, J. K. (1982). Pragmatic functions in aphasia. Journal of Communication Disorders, 15, 337-346

[7] Prinz, P. M. (1980). A note on requesting strategies in adult aphasia. Journal of Communication Disorders, 13, 65-73

[8] Gurland, G., Chwat, S., \& Wollner, S.G. (1982). Establishing a communication profile in adult aphasia: Analysis of communicative acts and conversational sequences. In R. H. Brookshire (Ed.), Clinical Aphasiology: Conference proceedings (pp. 18-27). Minneapolis, MN: BRK Publishers

[9] Prutting, C., \& Kirchner, D. (1987). A clinical appraisal of the pragmatic aspects of language. Journal of Speech and Hearing
Disorders, 52, 105-119

[10] Wilcox, M. J., and Davis, G. A. (1977). Speech act analysis of aphasic communication in individual and group settings. In R. H. Brookshire (Ed.), Clinical Aphasiology: Conference proceedings (pp.166-174). Minneapolis, MN: BRK Publishers

[11] Damico, J., Wilson, B., Simmons-Mackie, N. and Tetnowski, J. (2008). Overcoming Unintelligibility in Aphasia: The impact of non-verbal interactive strategies. Clinical Linguistics \& Phonetics, 22(10-11), 775-782

[12] Kertesz, A. (1982). The Western Aphasia Battery. New York: Grune \& Stratton

[13] Ripich, D. N., Vertes, D., Whitehouse, P., Fulton, S. \& Ekelman, B.(1991). Turn-taking and speech act patterns in the discourse of senile dementia of the Alzheimer's type patients. Brain and Language, 40, 330-343

[14] Doyle, P. J., Thompson, C. K., Oleyar, K., Wambaugh, J. \& Jackson, A. (1994). The effects of setting variables on conversational discourse in normal and aphasic adults. In R.H. Brookshire (Ed.), Clinical Aphasiology: Conference proceedings (pp. 135-144). Minneapolis, MN: BRK Publishers

[15] Wambaugh, J. L., Thompson, C. K., Doyle, P. J., \& Camarata, S. (1991). Conversational discourse of aphasic and normal adults: An analysis of communicative functions. In R. H. Brookshire (Ed.), Clinical Aphasiology: Conference proceedings (pp. 343-353). Minneapolis, MN: BRK Publishers

[16] Dore, J. (1978). Requestive systems in nursery school conversations: Analysis of talk in its social context. In R. Campbell \& P. Smith (Eds.), Recent Advances in the Psychology of Language: Language development and mother-Child interaction. New York: Plenum Press

[17] Behrmann, M., \& Penn, C. (1984). Non-verbal communication of aphasic patients. British Journal of Communication, $19,155-168$

[18] Kagan, A. \& Simmons-Mackie, N. (2007). Beginning with the end: Outcome-driven assessment and intervention with life participation in mind. Topics in Language Disorders, 27(4), p.309-317. 